\title{
Long non-coding RNA MALAT1 is up-regulated in ovarian cancer tissue and promotes SK-OV-3 cell proliferation and invasion
}

\author{
A. $\mathrm{ZOU}^{1, *}, \mathrm{R} . \mathrm{LIU}^{2, *}, \mathrm{X} . \mathrm{WU}^{3, *}$ \\ ${ }^{1}$ Department of Obstetrical, The Children \& Women's Healthcare of Laiwu City, Laiwu 271100, Shandong, China; ${ }^{2}$ People's Hospital of Zoucheng \\ City, Zoucheng 273500, Shandong, China; ${ }^{3}$ Department of Gynaecology II, Taian City Central Hospital, Taian 271000, Shandong, China
}

*Correspondence: wuxingguo778@126.com

${ }^{*}$ Contributed equally to this work.

\section{Received January 7, 2016 / Accepted May 17, 2016}

\begin{abstract}
Ovarian cancer is a gynecological malignancy worldwide. Long non-coding RNAs (lncRNAs) research is an emerging area in cancer studies, but little is known about lncRNA metastasis associated lung adenocarcinoma transcript 1 (MALAT1) in ovarian cancer. This study aims to investigate expression and roles of MALAT1 in ovarian cancer. MALAT1 level was detected in 20 ovarian cancer patients. MALAT1 expression was promoted by transforming growth factor $\beta 1$ (TGFB1) treatment and inhibited by siRNA transfection in human ovarian cancer cell line SK-OV-3, after which changes in cell viability, proliferation, migration and invasion were analyzed by MTT, colony formation and Transwell assays. Protein levels of mitogen-activated protein kinase factors, including MAPK kinase 1 (MEK1), extracellular signal-regulated kinase (ERK1), p38 and c-Jun N-terminal kinase 1 (JNK1), were detected by western blot. Results showed that MALAT1 was significantly up-regulated in ovarian cancer tissues compared to adjacent normal tissues $(P<0.001)$, and its expression was correlated to tumor size $\left(\mathrm{r}^{2}=0.7770, P<0.0001\right)$ and metastasis. TGFB1 and siRNA successfully altered MALAT1 levels in SK-OV-3 cells. Knockdown of MALAT1 suppressed SK-OV-3 cell viability, proliferation, migration and invasion $(P<0.05)$, and inhibited phosphorylation of MEK1, ERK1, p38 and JNK1, which suggested that MALAT1 promoted ovarian cancer cell proliferation, migration and invasion, and that MAPK pathways might be one of the regulatory mechanisms of MALAT1. This study reveals that MALAT1 is a potential biomarker for tumor growth and metastasis, as well as a promising therapeutic target in ovarian cancer, facilitating further ovarian cancer research.
\end{abstract}

Key words: ovarian cancer, MALAT1, proliferation, invasion, mitogen-activated protein kinase

Ovarian cancer is a gynecological malignancy with high morbidity and mortality. There are about 21,980 new cases and 14,270 deaths in USA in 2014 [1]. The majority of ovarian cancer is epithelial origin, and few cases are developed from sex-cord stromata, germ cells or other cell types [2], thus the ovarian epithelium is generally considered to be the primary location of ovarian cancer. A theory on ovarian cancer etiology is that incessant ovulation involving repetitive activities of the ovarian epithelium is a risk factor for ovarian cancer, which is supported by studies on oral contraceptives [3]. However, adverse side effects of oral contraceptives should be taken into consideration. Besides, no valid correlation has been found between ovarian cancer and the use of fertility drugs [4]. Surgery options, such as salpingectomy at the time of hysterectomy, are recommended to ovarian cancer patients $[5,6]$. But it remains a thorny problem to uncover the molecular mechanism of ovarian cancer and to find effective treatment strategy.

Given the molecular diversity and complexity of ovarian cancer, molecular research are necessary for the improvement in therapeutic outcomes [7]. microRNAs and other effective regulators of gene expression, as well as a number of vital genes and pathways, have become hotspots of ovarian cancer research in recent years [8]. Mitogen-activated protein kinase (MAPK) signaling, for instance, mainly includes extracellular signal-regulated kinase (ERK)/MAPK, p38 (alias MAPK14)/ MAPK and c-Jun N-terminal kinase (JNK)/MAPK pathways, is involved in modulating ovarian cancer cells [9]. Some very recent studies focus on the association of long non-coding RNAs (lncRNAs) ranging in length from $200 \mathrm{nt}$ to $100 \mathrm{~kb}$, which were previously considered as "dark matter" of the transcriptome. The functions of lncRNAs in regulating gene 
expression during human cancers are gradually uncovered [10], among which HOST2 and PVT1 participate in the development of ovarian cancer $[11,12]$.

Metastasis associated lung adenocarcinoma transcript 1 (MALAT1) is a lncRNA that relates to several types of cancers based on existed studies, and its overexpression is associated with unfavorable overall survival in non-small cell lung cancer, pancreatic cancer, gastric cancer and bladder cancer [13]. In the microarray analysis of lncRNAs, MALAT1 is aberrantly expressed in different ovarian cancer cell lines [14]. Still little is known about the role of MALAT1 in ovarian cancer. This study aims at investigating the expression and effects of MALAT1 in ovarian cancer. We detected MALAT1 expression in cancer and normal tissues of ovarian cancer patients and performed transforming growth factor $\beta 1$ (TGFB1) treatment and siRNA transfection to alter MALAT1 expression in human ovarian cancer cell line SK-OV-3. Cell „viability, colony formation, migration and invasion were analyzed. This study reveals MALAT1 as a promising target for ovarian cancer treatment, which will facilitate $\operatorname{lncRNA}$ research in ovarian cancer.

\section{Materials and methods}

Tissue sampling. The ovarian cancer and the adjacent normal tissue samples used in this study were collected from patients diagnosed of ovarian cancer and hospitalized from April 2014 to April 2015. The patients received surgical resection but not chemotherapy or radiotherapy. The patients were divided into two categories based on their in situ or metastasis ovarian cancer. Ten patients with in situ ovarian cancer aging from 30 to $67(48.2 \pm 4.1)$ and ten patients with metastasis ovarian cancer aging from 27 to $69(50.5 \pm 4.5)$ were randomly chosen from each category, with no significant difference between the age of the two groups $(P>0.05)$. The tumor size was measured. The cancer and normal tissue samples were immediately stored at $-80^{\circ} \mathrm{C}$ for RNA extraction. This procedure was approved by the research ethics committee of the hospital and the patients were informed before tissue sampling.

Cell culture. Human ovarian cancer cell line SK-OV-3 (ATCC, Manassas, VA) was cultured in Roswell Park Memorial Institute-1640 medium supplemented with $10 \%$ fetal bovine serum (FBS), $100 \mathrm{U} / \mathrm{mL}$ penicillin and $100 \mu \mathrm{g} / \mathrm{mL}$ streptomycin (Gibco, Carlsbad, CA). The cells were incubated in humidified atmosphere with $5 \% \mathrm{CO}_{2}$ at $37^{\circ} \mathrm{C}$ [15]. The medium was changed every three days.

TGFB1 treatment and siRNA transfection. The cultured cells were divided into four groups: TGFB1 (cells treated with TGFB1 to promote MALAT1), blank control (cells as the control for TGFB1 treatment), si-MALAT1 (cells transfected with MALAT1 siRNA), and si control (cells as the control for MALAT1 knockdown).

TGFB1 was used in this study to promote MALAT1 expression according to a previous study [16]: The TGFB1 human recombinant protein (Peprotech, Rocky Hill, NJ) was dissolved in $10 \mathrm{mM}$ citric acid solution (pH 3.0) and added in TGFB1 group to a final concentration of $0.5 \mathrm{ng} / \mathrm{mL}$. The cells were treated by TGFB1 for $36 \mathrm{~h}$ until the following experiments. For blank control group, the same amount of citric acid solution was added to the cells for the same treating duration.

The MALAT1-specific siRNA (Sigma-Aldrich, Shanghai, China) was transfected to SK-OV-3 cells of si-MALAT1 group by Lipofectamine RNAiMAX (Invitrogen, Carlsbad, CA) according to the manufacturer's instruction. Briefly, the cells in the logarithmic phase were seeded in 6-well plates at $24 \mathrm{~h}$ before transfection. The confluence of cells reached $90 \%$ and serum-free medium was used for transfection. At $6 \mathrm{~h}$ after transfection, the medium was changed into complete medium. The cells of si control group were transfected with scramble siRNA (Sigma-Aldrich) as a control.

Cell viability assay. Cell viability was measured by $3-(4,5-$ dimethylthiazol-2-yl)-2,5-diphenyltetrazolium bromide (MTT) method according to a previous study [17]. Cells in the logarithmic phase of each group were seeded in 96 -well plates $\left(1 \times 10^{4}\right.$ cells/well $)$ and cultured in humidified atmosphere with $5 \% \mathrm{CO}_{2}$ at $37^{\circ} \mathrm{C}$. At 0,24 or $48 \mathrm{~h}$ after transfection, $20 \mu \mathrm{L}$ of $0.5 \%$ MTT (Sigma-Aldrich) was added to each well and the cells were incubated for another $4 \mathrm{~h}$. Then the medium was discarded and $100 \mu \mathrm{L}$ of dimethyl sulfoxide was added, and the plates were shaken gently to dissolve formazan. The optical density at $570 \mathrm{~nm}$ was detected with a microplate reader (Bio-Rad, Hercules, CA).

Colony formation assay. Colony formation assay was performed to assess the proliferation ability of SK-OV-3 cells. Cells in the logarithmic phase were digested to single-cell suspension by trypsin (Sigma-Aldrich). The cell suspension containing 200 cells were seeded in 6 -cm culture dishes with complete medium, and the plates were incubated in humidified atmosphere with $5 \% \mathrm{CO}_{2}$ at $37^{\circ} \mathrm{C}$ for two weeks. Then the medium was removed and the plates were washed gently by phosphate buffered saline (PBS) twice, after which the cells were fixed by methanol for $15 \mathrm{~min}$ and stained in Giemsa (HarveyBio, Beijing, China) for $20 \mathrm{~min}$. After washed, the cells were air-dried, and colony number of each group was calculated.

Transwell assay. At $48 \mathrm{~h}$ after TGFB1 treatment or siMALAT1 transfection, the cells were used for Transwell assay. For cell invasion assay, the upper chamber of Transwell (pore diameter $=8 \mu \mathrm{m}, \mathrm{BD}$ Biosciences, San Jose, CA) was pre-coated with Matrigel (BD Biosciences) and incubated at $37^{\circ} \mathrm{C}$ for $30 \mathrm{~min}$ for gel formation, after which the gel was infiltrated by serum-free medium for $2 \mathrm{~h}$. The cells were digested and resuspended in serum-free medium to a density of $5 \times 10^{5}$ cells $/ \mathrm{mL}$, and $100 \mu \mathrm{L}$ of the cell suspension was seeded in the upper chamber. The lower chamber was filled with medium containing $20 \% \mathrm{FBS}$, and then the chambers were incubated at $37^{\circ} \mathrm{C}$ for $24 \mathrm{~h}$. After the incubation, the medium of the upper chamber was removed, and the cells remained in the upper chamber were wiped out with a cotton swab and washed with PBS. The cells on the lower side of the membrane were stained with crystal violet (Beyotime, 
Shanghai, China) for $20 \mathrm{~min}$ and counted under an optical microscope (Olympus, Tokyo, Japan). For cell viability assay, the same procedures were performed but without the use of Matrigel.

qRT-PCR. The tissue or cell samples were lysed and RNA was extracted by Trizol (Invitrogen) according the manufacturer's instruction. Protein and DNA contamination in RNA samples was eliminated by RNA Purification Kit (TIANGEN, Beijing, China). Total RNA (1 $\mu \mathrm{g})$ was used for the complementary DNA synthesis under the catalysis of PrimeScript Reverse Transcriptase (Takara, Dalian, China). The relative expression level of MALAT1 in tissues were detected on the LightCycler 480 platform (Roche, Basel, Switzerland) with the specific primer for human MALAT1 (Fw: 5'-AAA GCA AGG TCT CCC CAC AAG-3' and Rv: 5'-GGT CTG TGC TAG ATC AAA AGG CA-3') and Gapdh (Fw: 5'-GAA GGT GAA GGT CGG AGT C-3' and Rv: 5'-GAA GAT GGT GAT GGG ATT TG-3') as an internal control [17]. Data were analyzed by the $2^{-\Delta \Delta C t}$ method.

Western blot. Protein from the cell lysate was extracted using Radio Immunoprecipitation Assay Lysis Buffer (Beyotime, Shanghai, China) according to the manufacturer's instructions. Western blot was performed as previously described [15]. The protein samples were separated by sodium dodecyl sulfate-polyacrylamide gel electrophoresis and blotted to a polyvinylidene fluoride membrane. The blot was blocked in $5 \%$ skim milk for $2 \mathrm{~h}$ at room temperature and then incubated in the specific primary antibodies for the factors and their phosphorylation forms in MAPK pathways (ab32091, ab96379, ab32537, ab131438, ab170099, ab45381, ab199380, ab47337, abcam, Cambridge, UK) at $4^{\circ} \mathrm{C}$ overnight. GAPDH (ab9484) was used as an internal control. After washed in PBS, the blot was incubated in horse reddish peroxidase-conjugated secondary antibodies (abcam) for $2 \mathrm{~h}$ at room temperature. Signals were developed by ECL Plus Western Blotting Substrate (Pierce, Carlsbad, CA) and the grayscale of each band relative to GAPDH was compared.
Statistical analysis. All the experiments were performed in triplicates, and results were represented as the mean \pm standard deviation and analyzed by SPSS 19 . Pearson's correlation was used to analyze the correlation between MALAT1 expression and tumor size. The other data were analyzed using $F$ test for homogeneity of variance and then $t$ test for statistical difference. The difference between groups was considered significant when $P<0.05$.

\section{Results}

MALAT1 is elevated in ovarian cancer tissues. First of all, we compared MALAT1 expression in cancer and normal tissues of ovarian cancer patients by qRT-PCR (Figure 1A), and results showed an obviously elevated level of MALAT1 in cancer tissues than normal tissues $(P<0.001)$, which might imply the potential function of MALAT1 in ovarian cancer cells. Then we associated MALAT1 level with the tumor size of each patient and found that MALAT1 expression was in positive correlation to tumor size: patients with larger tumor sizes tended to possess higher MALAT1 expression levels $\left(\mathrm{r}^{2}\right.$ $=0.7770, P<0.0001$, Figure 1B). We also compared MALAT1 expression between metastasis cancer and in situ cancer, and results indicated that MALAT1 was significantly higher expressed in metastasis cancer tissues than in situ cancer tissues $(P<0.001$, Figure 1C). These results implied that MALAT1 expression level was likely to be related to tumor growth and metastasis of ovarian cancer, so we next examined the influence of MALAT1 on ovarian cancer cell capacities in SK-OV-3 cells.

MALAT1 promotes proliferation, migration and invasion of ovarian cancer cells. In order to examine the role of MALAT1 in SK-OV-3 cells, we overexpressed MALAT1 by TGFB1 treatment and inhibited MALAT1 by siRNA transfection. Results showed that TGFB1 treatment in SK-OV-3 cells significantly promoted MALAT1 expression $(P<0.01)$ and si-MALAT1 inhibited MALAT1 expression $(P<0.001$, Figure $1 \mathrm{~A})$. So the
A

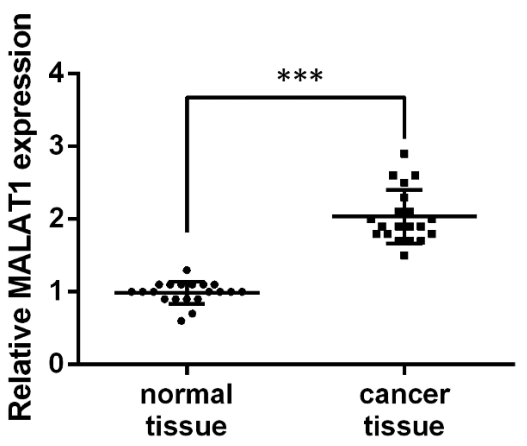

B

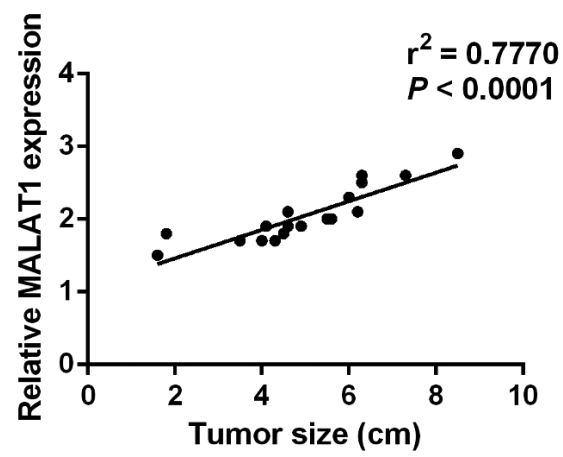

C

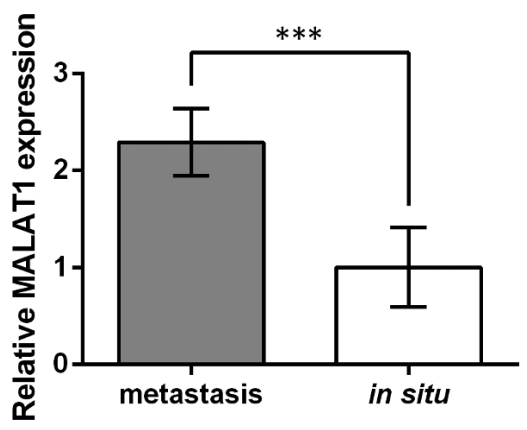

Figure 1. MALAT1 is elevated in ovarian cancer tissues and is associated with tumor size and cancer metastasis. (A) qRT-PCR detects MALAT1 level in ovarian cancer tissues compared to normal tissues in 20 patients. GAPDH is an internal control. (B) Positive correlation between MALAT1 expression and the tumor size of 20 patients. (C) MALAT1 level is significantly lower in the 10 patients with in situ cancer than those with metastasis cancer. ${ }^{* *}$ $P<0.001$. MALAT1, metastasis associated lung adenocarcinoma transcript 1 . 
two methods could effectively alter MALAT1 level, and the four cell groups were used in the following experiments.

Viability and proliferation of SK-OV-3 cells were analyzed by MTT and colony formation assay, respectively. MTT results showed that the viability of TGFB1-treated cells was obviously promoted when detected at 24 and $48 \mathrm{~h}$ after treatment $(P<0.05$, Figure $2 \mathrm{~B})$, and si-MALAT1 had the opposite influence that significantly inhibited cell viability at 24 and 48 $\mathrm{h}$ after transfection $(P<0.05$, Figure $2 \mathrm{C})$. Colony formation assay showed similar results: TGFB1-treated cells formed more colonies than blank control, and si-MALAT1 transfection reduced colony number compared to si control (Figure 2D). Significant changes were detected when replicated experiments were analyzed $(P<0.01$, Figure $2 \mathrm{E})$. Taken together, these results indicated that knockdown of MALAT1 suppressed cell SK-OV-3 cell viability and proliferation, and that TGFB1-induced MALAT1 overexpression was accompanied by promoted cell viability and proliferation.
Then we analyzed SK-OV-3 cell migration and invasion using Transwell assay. Cell migration results showed that the migrated cells in TGFB1 group were more than blank control, while those in si-MALAT1 group were less than si control (Figure 3A). Similar results were acquired in replicated experiments, with significant differences between groups $(P<$ 0.001 or $P<0.01$, Figure 3B). Moreover, TGFB1 treatment induced cell invasion, while si-MALAT1 transfection inhibited cell invasion (Figure 3C), both showing significant difference compared to the corresponding control group $(P<0.01$ or $P<0.05$, Figure 3D). Taken together, knockdown of MALAT1 inhibited SK-OV-3 cell migration and invasion, and TGFB1induced MALAT1 overexpression was accompanied by these promoted cell abilities.

MALAT1 activated MAPK pathways. Next we detected the expression of four factors in MAPK pathways and their phosphorylation forms by western blot to analyze the effects of MALAT1 on MAPK pathways. Results showed that the
A

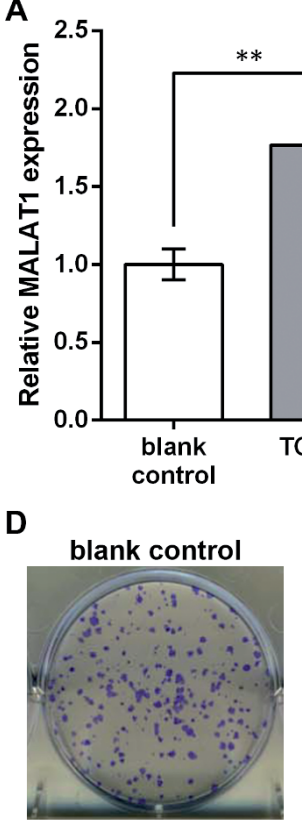

si control

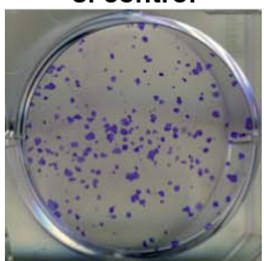

B

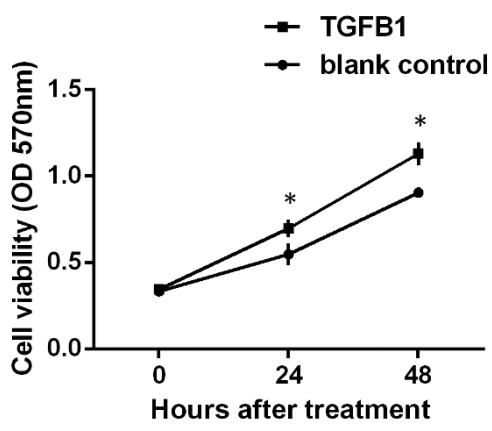

C

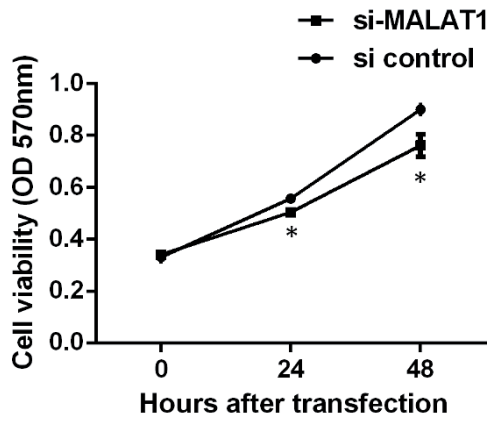

Figure 2. MALAT1 promotes SK-OV-3 cell viability and proliferation. (A) MALAT1 level is effectively inhibited by TGFB1 treatment and promoted by si-MALAT1 in SK-OV-3 cells $(\mathbf{n}=3)$. GAPDH is an internal control. $(B)$ MTT assay shows elevated viability in cells with promoted MALAT1 $(n=3)$. (C) MTT assay shows suppressed viability in cells with inhibited MALAT1 $(n=3)$. Cell viability was detected at $0,24,48 \mathrm{~h}$ after treatment at an optical density of $570 \mathrm{~nm}$. (C) Pictures of colony formation assay of the four groups. Cells of TGFB1 group formed more colonies than blank control, and cells of si-MALAT1 groups formed fewer colonies than si control when observed after two weeks of incubation. (D) Histogram indicating the colony number of each group based on replicated colony formation assay $(n=3) .{ }^{*}<<0.05$. ${ }^{*} P<0.01$. ${ }^{\star * *} P<0.001$. (E) MALAT1, metastasis associated lung adenocarcinoma transcript 1. TGFB1: transforming growth factor $\beta 1$. 
total protein level of MAPK kinase 1 (MEK1, alias MAP2K1), ERK1 (alias MAPK3), p38 or JNK1 was hardly changed by TGFB1 treatment or si-MALAT1 transfection in SK-OV-3 cells (Figure 4). However, the phosphorylation forms of the four proteins, namely, p-MEK1, p-ERK1, p-p38 and p-JNK1, were all up-regulated by TGFB1 and down-regulated by siMALAT1 compared to control groups. Based on these results, knockdown of MALAT1 was able to inhibit the activation of the four MAPK factors, and TGFB1-induced MALAT1 overexpression was accompanied by activation of these factors.

\section{Discussion}

As research hotspots in recent year, lncRNAs and their pivotal roles in gene regulation and disease control have received considerable attention. MALAT1 is considered to be a paradigm for lncRNAs in cancer research [18]. We perform a preliminary study on MALAT1 of its expression and roles in ovarian cancer. In vivo MALAT1 level is up-regulated in ovarian cancer tissues. Knockdown of MALAT1 in human ovarian cancer cell line SK-OV-3 suppresses cell viability, proliferation, migration and invasion, as well as the activation of MAPK factors such as MEK1, ERK1, p38 and JNK1.

Based on the results of existed studies, MALAT1 is generally overexpressed in cancer or lesion tissues of pancreatic cancer [19], unstable angina [20], colorectal cancer [21], glioma cancer [22], amongst others. In pancreatic duct adenocarcinoma, MALAT1 is positively correlated to tumor size, tumor stage, depth of invasion and survival rate of patients [19]. High MALAT1 expression is also correlated with tumor size, tumor stage and lymph node metastasis in cervical cancer [23], esophageal squamous cell carcinoma [24] and many other cancers [25], which suggests MALAT1 as a potential biomarker of tumor development and metastasis. In this study
A

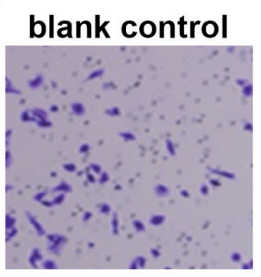

si control

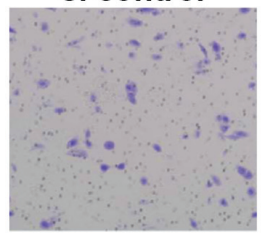

C

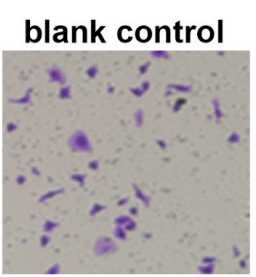

si control

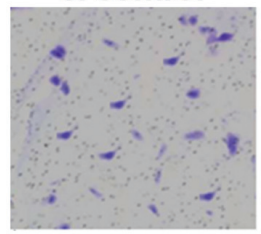

TGFB1

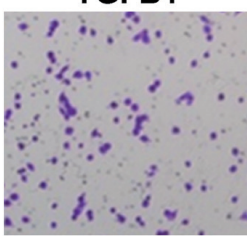

si-MALAT1

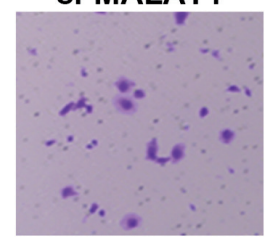

TGFB1

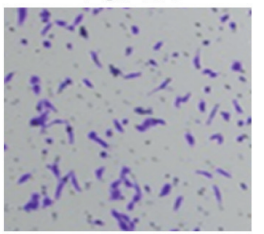

si-MALAT1

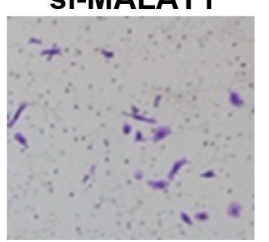

B

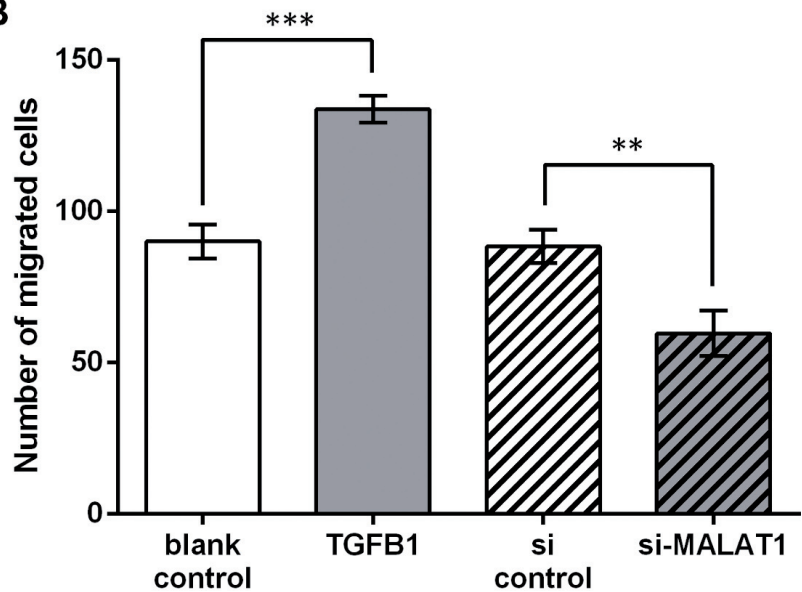

D

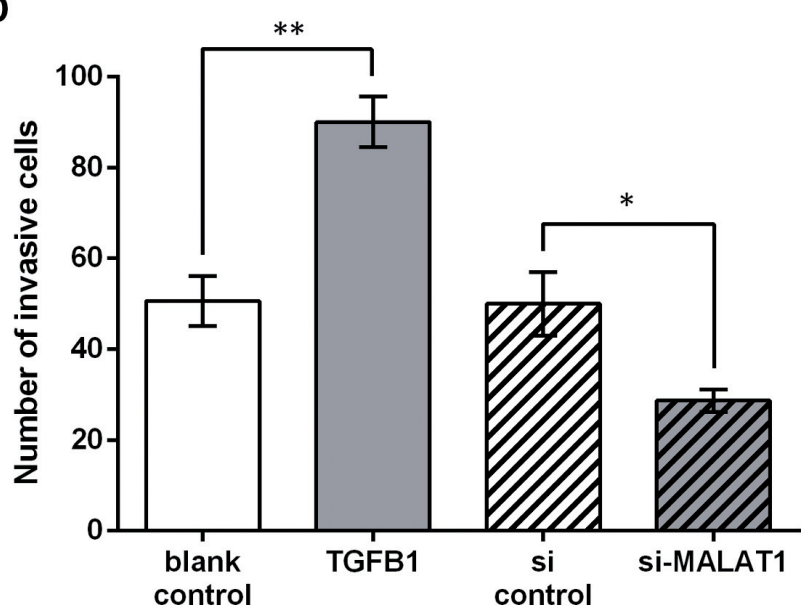

Figure 3 MALAT1 promotes SK-OV-3 cell migration and invasion. (A) Pictures of migrated cells detected by Transwell assay at $48 \mathrm{~h}$ after TGFB1 treatment or si-MA migrated cells. (B) Histogram of migrated cell number based on replicated experiments $(n=3)$. (C) Pictures of invasive cells detected by Transwell assay with Matrigel at $48 \mathrm{~h}$ after TGFB1 treatment or si-MA migrated cells.. (D) Histogram of invasive cell number based on replicated experiments $(\mathrm{n}=3) .{ }^{\star} P<0.05 .{ }^{* *} P<0.01 .{ }^{* *} P<0.001$. (D) MALAT1, metastasis associated lung adenocarcinoma transcript 1 . TGFB1: transforming growth factor $\beta 1$. 


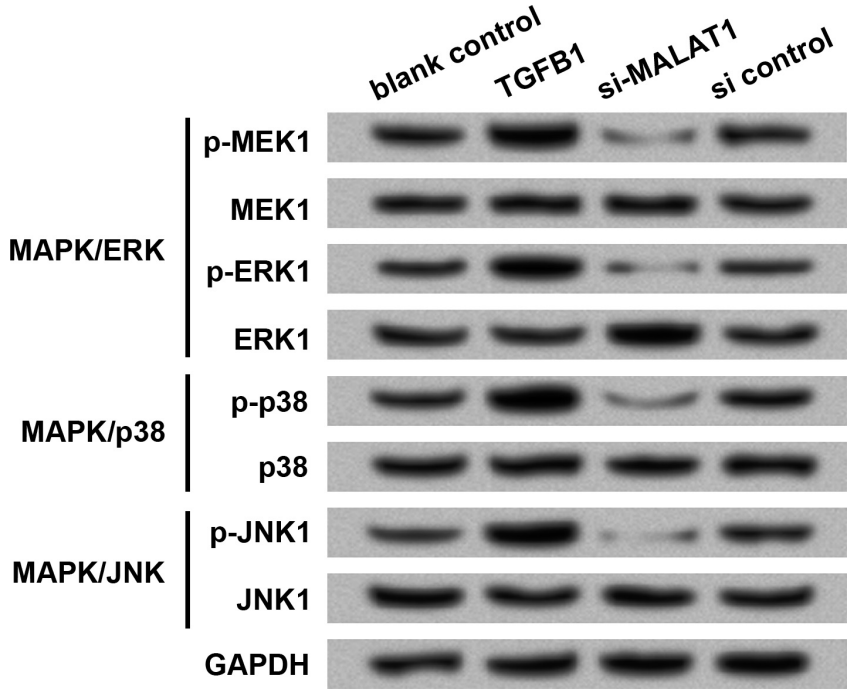

Figure 4. Protein levels of MAPK factors in SK-OV-3 cells. Western blot is performed at $48 \mathrm{~h}$ after treatment or transfection. GAPDH is an internal control. Levels of MEK1, ERK1, p38 and JNK1 are hardly changed, while their phosphorylation forms are altered by TGFB1 treatment or si-MALAT1 transfection. MALAT1, metastasis associated lung adenocarcinoma transcript 1. TGFB1: transforming growth factor $\beta 1$. MAPK, mitogen-activated protein kinase. MEK1, MAPK kinase 1 (MAP2K1). ERK1, extracellular signal-regulated kinase 1 (MAPK3). P38, MAPK14. JNK1, c-Jun N-terminal kinase 1.

on ovarian cancer, MALAT1 was significantly up-regulated in cancer tissues compared to normal tissues, and its expression was in positive correlation to tumor size. Besides, higher MALAT1 level was detected in patients with cancer metastasis. These findings are in consistent with the previous studies on MALAT1, and imply that MALAT1 may be a promising biomarker for tumor growth and metastasis in ovarian cancer.

In multiple myeloma, MALAT1 is capable of promoting the binding of transcription factor Sp1 to latent TGFB binding protein 3 (LTBP3) which regulates the activity of TGFBs [26]. A study in bladder cancer further suggests that TGFB1 promotes MALAT1 expression and epithelial-mesenchymal transition (EMT) [16], which inspired us to use TGFB1 treatment for the positive regulation of MALAT1 in human ovarian cancer cell line SK-OV-3. As expected, TGFB1 treatment successfully induced up-regulation of MALAT1 in SK-OV-3 cells which were then used together with siMALAT1-transfected cells for cell capacity assays. Though inverse changing patterns were observed between TGFB1 and si-MALAT groups, it was not certain whether the effect of TGFB1 treatment was purely dependent on the induced MALAT1 overexpression, without affecting other factors. So the results of TGFB1 treatment were considered as a complementary for altered MALAT1 expression.

Previous studies have discussed the relatively conserved functions of MALAT1 in promoting cell proliferation, migration and invasion. MALAT1 induces hematopoietic cell proliferation and helps to maintain the undifferentiated status [27]. It also promotes cell proliferation, migration and invasion, and facilitates tumor metastasis in lung cancer [28], osteosarcoma [29], cervical cancer [23], clear cell kidney carcinoma [30], breast cancer [31] and esophageal squamous cell carcinoma [24], thus being a potential target for cancer treatment. Knockdown of MALAT1 in this study suppressed SK-OV-3 cell viability, proliferation, migration and invasion, while TGFB1-induced MALAT1 was associated with promotion in these cell capacities, which implies the pro-proliferation, migration and invasion roles of MALAT1 in ovarian cancer cells. So the function of MALAT1 is conserved in these reported diseases, and MALAT1 is a promising therapeutic target for ovarian cancer treatment.

It is reported that MALAT1 participates in cancer cell modulation via regulating EMT $[32,33]$ and cancer-related downstream pathways including PI3K/AKT [29], miR-200s/ ZEB2 [30], ATM/CHK2 [24] and EZH2 [34]. In this study, knockdown of MALAT1 inhibited the activation of MAPK factors MEK1, ERK1, p38 and JNK1, and MALAT1 overexpression might induce phosphorylation of these factors. MAPK signaling, which mainly consists of MEK/ERK/MAPK, p38/MAPK and JNK/MAPK, is involved in cancer cell proliferation, migration and invasion [35-37]. This study suggests the three major MAPK pathways are all regulated by MALAT1 in ovarian cancer, which may be one of the functional mechanisms of MALAT1 in cancer modulation. Future studies will focus on the direct association between MALAT1-induced MAPK activation and ovarian cancer cell capacities.

\section{Conclusion}

In summary, this study reveals the up-regulation of MALAT1 in ovarian cancer tissues and the promotive effects of MALAT1 on SK-OV-3 cell proliferation, migration and invasion. MALAT1 is a promising biomarker for tumor growth and metastasis for ovarian cancer, as well as a potential therapeutic target for cancer treatment. More efforts will be made to investigate the regulatory mechanism of MALAT1 in ovarian cancer.

\section{References}

[1] SIEGEL R, MA J, ZOU Z, JEMAL A. Cancer statistics, 2014. CA Cancer J Clin 2014; 64: 9-29. http://dx.doi.org/10.3322/ Caac. 21208

[2] KALIR T, FIRPO-BETANCOURT A, NEZHAT F. Update on ovarian cancer pathogenesis: history, controversies, emerging issues and future impact. Expert Rev Obstet Gynecol 2013; 8: 539-547. http://dx.doi.org/10.1586/17474108.2013.847638

[3] FATHALLA MF. Incessant Ovulation - a Factor in Ovarian Neoplasia? The Lancet 1971; 298: 163. http://dx.doi. org/10.1016/S0140-6736(71)92335-X

[4] FATHALLA MF. Incessant ovulation and ovarian cancer - a hypothesis re-visited. Facts Views Vis Obgyn 2013; 5: 292-297. 
[5] NEZHAT FR, APOSTOL R, NEZHAT C, PEJOVIC T. New insights in the pathophysiology of ovarian cancer and implications for screening and prevention. Am J Obstet Gynecol 2015; 213: 262-267. http://dx.doi.org/10.1016/j. ajog.2015.03.044

[6] LI Y. The possible pathology and pharmaceutical therapy of ovarian cancer. Eur J BioMed Res 2016; 2: 32-35. http://dx.doi. org/10.18088/ejbmr.2.1.2016.pp32-35

[7] LIU J, MATULONIS UA. New strategies in ovarian cancer: translating the molecular complexity of ovarian cancer into treatment advances. Clin Cancer Res 2014; 20: 5150-5156. http://dx.doi.org/10.1158/1078-0432.CCR-14-1312

[8] KINOSE Y, SAWADA K, NAKAMURA K, KIMURA T. The role of microRNAs in ovarian cancer. Biomed Res Int 2014; 2014: 249393. http://dx.doi.org/10.1155/2014/249393

[9] CHANG MC, CHEN CA, CHEN PJ, CHIANG YC, CHEN YL et al. Mesothelin enhances invasion of ovarian cancer by inducing MMP-7 through MAPK/ERK and JNK pathways. Biochem J 2012; 442: 293-302. http://dx.doi.org/10.1042/ B]20110282

[10] GIBB EA, BROWN CJ, LAM WL. The functional role of long non-coding RNA in human carcinomas. Mol Cancer 2011; 10: 38-54. http://dx.doi.org/10.1186/1476-4598-10-38

[11] LIU E, LIU Z, ZHOU Y. Carboplatin-docetaxel-induced activity against ovarian cancer is dependent on up-regulated lncRNA PVT1. Int J Clin Exp Pathol 2015; 8: 3803-3810.

[12] GAO Y, MENG H, LIU S, HU J, ZHANG Y et al. LncRNAHOST2 regulates cell biological behaviors in epithelial ovarian cancer through a mechanism involving microRNA let-7b. Hum Mol Genet 2015; 24: 841-852. http://dx.doi. org/10.1093/hmg/ddu502

[13] ZHANG J, ZHANG B, WANG T, WANG H. LncRNA MALAT1 overexpression is an unfavorable prognostic factor in human cancer: evidence from a meta-analysis. Int J Clin Exp Med 2015; 8: 5499-5505.

[14] LIU SP, YANG JX, CAO DY, SHEN K. Identification of differentially expressed long non-coding RNAs in human ovarian cancer cells with different metastatic potentials. Cancer Biol Med 2013; 10: 138-141.

[15] LI Y, WANG K, JIANG YZ, CHANG XW, DAI CF et al. 2,3,7,8-Tetrachlorodibenzo-p-dioxin (TCDD) inhibits human ovarian cancer cell proliferation. Cell Oncol (Dordr) 2014; 37: 429-437. http://dx.doi.org/10.1007/s13402-014-0206-4

[16] FAN Y, SHEN B, TAN M, MU X, QIN Y et al. TGF-betainduced upregulation of malat1 promotes bladder cancer metastasis by associating with suz12. Clin Cancer Res 2014; 20: 1531-1541. http://dx.doi.org/10.1158/1078-0432.CCR$\underline{13-1455}$

[17] LI Y, WANG K, ZOU QY, MAGNESS RR, ZHENG J. 2,3,7,8Tetrachlorodibenzo-p-dioxin differentially suppresses angiogenic responses in human placental vein and artery endothelial cells. Toxicology 2015; 336: 70-78. http://dx.doi. org/10.1016/j.tox.2015.08.003

[18] GUTSCHNER T, H MMERLE M, DIEDERICHS S. MALAT1 - a paradigm for long noncoding RNA function in cancer. J Mol Med (Berl) 2013; 91: 791-801. http://dx.doi.org/10.1007/ $\underline{\text { s00109-013-1028-y }}$
[19] LIU J-H, CHEN G, DANG Y-W, LI C-J, LUO D-Z. Expression and Prognostic Significance of lncRNA MALAT1 in Pancreatic Cancer Tissues. Asian Pac J Cancer Prev 2014; 15: 2971-2977. http://dx.doi.org/10.7314/ APJCP.2014.15.7.2971

[20] TANG Y, JIN X, XIANG Y, CHEN Y, SHEN CX et al. The IncRNA MALAT1 protects the endothelium against oxLDL-induced dysfunction via upregulating the expression of the miR-22-3p target genes CXCR2 and AKT. FEBS Lett 2015; 589: 3189-3196. http://dx.doi.org/10.1016/j. febslet.2015.08.046

[21] ZHENG HT, SHI DB, WANG YW, LI XX, XU Y et al. High expression of $\operatorname{lncRNA}$ MALAT1 suggests a biomarker of poor prognosis in colorectal cancer. Int J Clin Exp Pathol 2014; 7: 3174-3181.

[22] MA KX, WANG HJ, LI XR, LI T, SU G et al. Long noncoding RNA MALAT1 associates with the malignant status and poor prognosis in glioma. Tumour Biol 2015; 36: 3355-3359. http:// dx.doi.org/10.1007/s13277-014-2969-7

[23] YANG L, BAI HS, DENG Y, FAN L. High MALAT1 expression predicts a poor prognosis of cervical cancer and promotes cancer cell growth and invasion. Eur Rev Med Pharmacol Sci 2015; 19: 3187-3193.

[24] HU L, WU Y, TAN D, MENG H, WANG K et al. Upregulation of long noncoding RNA MALAT1 contributes to proliferation and metastasis in esophageal squamous cell carcinoma. J Exp Clin Cancer Res 2015; 34: 7. http://dx.doi. org/10.1186/s13046-015-0123-Z

[25] ZHAI H, CHEN QJ, CHEN BD, YANG YN, MA YT ET AL. Long noncoding RNA MALAT1 as a putative biomarker of lymph node metastasis: a meta-analysis. Int J Clin Exp Med 2015; 8: 7648-7654.

[26] LI B, CHEN P, QU J, SHI L, ZHUANG W et al. Activation of LTBP3 gene by a long noncoding RNA (lncRNA) MALAT1 transcript in mesenchymal stem cells from multiple myeloma. J Biol Chem 2014; 289: 29365-29375. http://dx.doi. org/10.1074/jbc.M114.572693

[27] MA XY, WANG JH, WANG JL, MA CX, WANG XC et al. Malat1 as an evolutionarily conserved lncRNA, plays a positive role in regulating proliferation and maintaining undifferentiated status of early-stage hematopoietic cells. BMC Genomics 2015; 16: 676. http://dx.doi.org/10.1186/ $\underline{\text { s12864-015-1881-X }}$

[28] GUTSCHNER T, H MMERLE M, EI MANN M, HSU J, KIM $\mathrm{Y}$ et al. The non-coding RNA MALAT1 is a critical regulator of the metastasis phenotype of lung cancer cells Cancer Res 2013; 73: 1180-1189. http://dx.doi.org/10.1158/0008-5472. CAN-12-2850

[29] DONG Y, LIANG G, YUAN B, YANG C, GAO R et al. MALAT1 promotes the proliferation and metastasis of osteosarcoma cells by activating the PI3K/Akt pathway. Tumour Biol 2015; 36: 1477-1486. http://dx.doi.org/10.1007/s13277014-2631-4

[30] XIAO H, TANG K, LIU P, CHEN K, HU J et al. LncRNA MALAT1 functions as a competing endogenous RNA to regulate ZEB2 expression by sponging miR-200s in clear cell kidney carcinoma. Oncotarget 2015; 6: 38005-38015. 
[31] LIU R, LI J, LAI Y, LIAO Y, LIU R et al. Hsa-miR-1 suppresses breast cancer development by down-regulating K-ras and long non-coding RNA MALAT1. Int J Biol Macromol 2015; 81: 491-497. http://dx.doi.org/10.1016/j.ijbiomac.2015.08.016

[32] SHEN L, CHEN L, WANG Y, JIANG Y, XIA H et al. Long noncoding RNA MALAT1 promotes brain metastasis by inducing epithelial-mesenchymal transition in lung cancer. J Neurooncol 2015; 121: 101-108. http://dx.doi.org/10.1007/ s11060-014-1613-0

[33] XU S, SUI S, ZHANG J, BAI N, SHI Q et al. Downregulation of long noncoding RNA MALAT1 induces epithelial-tomesenchymal transition via the PI3K-AKT pathway in breast cancer. Int J Clin Exp Pathol 2015; 8: 4881-4891.

[34] WANG D, DING L, WANG L, ZHAO Y, SUN Z et al. LncRNA MALAT1 enhances oncogenic activities of EZH2 in castration-resistant prostate cancer. Oncotarget 2015; 6: 41045-41055.

[35] ERLICH RB, KAHN SA, LIMA FR, MURAS AG, MARTINS RA et al. STI1 promotes glioma proliferation through MAPK and PI3K pathways. Glia 2007; 55: 1690-1698. http://dx.doi. org/10.1002/glia.20579

[36] TESSER-GAMBA F, LOPES LJ, PETRILLI AS, TOLEDO SR. MAPK7 gene controls proliferation, migration and cell invasion in osteosarcoma. Mol Carcinog 2015; [ahead of print] http://dx.doi.org/10.1002/mc.22420

[37] KIM EK, CHOI E-J. Pathological roles of MAPK signaling pathways in human diseases. Biochim Biophys Acta 2010; 1802: 396-405. http://dx.doi.org/10.1016/j. bbadis.2009.12.009 